\title{
Smart Inverter Impacts on California Distribution Feeders with Increasing PV Penetration: A Case Study
}

\author{
Zachary K. Pecenak \\ Center for Energy Research \\ University of California San Diego \\ La Jolla, California 92093 \\ Email: zpecenak@ucsd.edu
}

\author{
Jan Kleissl \\ Jacobs school of Engineering \\ University of California San Diego \\ La Jolla, California 92093 \\ Email: jkleiss1@ucsd.edu
}

\author{
Vahid Rasouli Disfani \\ Center for Energy Research \\ University of California San Diego \\ La Jolla, California 92093 \\ Email:vrdisfani@ucsd.edu
}

\begin{abstract}
The impacts of high PV penetration on distribution feeders have been well documented within the last decade. To mitigate these impacts, interconnection standards have been amended to allow PV inverters to regulate voltage locally. However, there is a deficiency of literature discussing how these inverters will behave on real feeders under increasing PV penetration. In this paper, we simulate several deployment scenarios of these inverters on a real California distribution feeder. We show that minimum and maximum voltage, tap operations, and voltage variability are improved due to the inverters. Line losses were shown to increase at high $P V$ penetrations as a side effect. Furthermore, we find inverter sizing was shown to be important as PV penetration increased. Finally we show that increasing the number of inverters and removing the deadband from the Volt/VAr control curve improves the effectiveness.
\end{abstract}

\section{INTRODUCTION}

The negative impacts of high PV penetration on electrical grids are well documented [1]. In distribution systems, rooftop PV can cause large voltage fluctuations, over-voltages, and increased activation and maintenance costs for voltage regulators. Over-voltages exceeding the mandated ANSI C84.1 voltage limits of $\pm 5 \%$ [2] pose a threat to behind-the-meter equipment. These problems become increasingly severe with increasing PV penetration [3].

In order to combat the negative effects of PV, both IEEE 1547 [4] and CA rule 21 [5] interconnection standards have been amended to allow the local regulation of voltage through real and reactive power modulation. Given that PV inverters already function to regulate the real power injected into the grid from the PV, they are a natural choice to function as the control agent. These inverters which act to modulate electrical bus real and reactive power (as well as several other advanced functions [6]), are hereafter referred to smart inverters (SI).

Power factor control (PFC) is one control method in which the power factor of the inverter operated on a fixed or variable schedule to regulate voltage. Volt/VAr control (VVC) is one of the functionalities of SI which follows some predetermined Volt/VAr curves. Typically VVC employs a negative sloped curve that instructs reactive power injection for low voltage and reactive power absorption for high voltage. Typical
Volt/VAr curves are shown in figure 2

[7] introduces 7 types of inverter voltage control schemes, 3 of which are PFC while the other 4 are VVC. Through a simple simulation they conclude that VVC improves voltage conditions for PV penetrations of 20\%. [8] showed that increased voltages and voltage fluctuations due to $\mathrm{PV}$ on a feeder can be damped by both PFC and VVC, with VVC being more effective.

[9] investigated the impact of local VVC on hosting capacity, which they define as the upper limit of PV penetration that does not cause violations in the network operating standards (i.e. ANSI range A limits). Their simulations on 6 feeders show that VVC can improve feeder hosting capacity, where the effectiveness increases with increasing inverter size.

[10] simulated a feeder with a number of VVC settings to determine which settings are the most appropriate. The authors quantify improvements in voltage, tap operations, line losses, and voltage fluctuations in terms of droop control settings and substation voltage. However, the one PV penetration considered was not specified in the paper. The authors conclude that optimal settings are feeder specific.

Most recently, [11] simulated the $\mathrm{J} 1$ feeder using realistic load and PV generation data at 15-min resolution to look at effects of Volt/VAr control on feeder voltages. The authors show that SI using VVC are capable of reducing maximum voltages on the feeder due to increasing PV production. The PV penetration was not specified by the authors.

To date, literature is lacking any discussion of SI operating under extremely high PV penetrations (PV penetration higher than 50\%) where adverse effects are greatest. Furthermore, all works assume that $100 \%$ of PV systems have VVC capabilities, which is unlikely considering already high PV penetrations in several states such as California and the high cost of retrofitting existing systems. In this work, we systematically quantify the SI effects at PV penetration up to $200 \%$ and the effects of different fractions of SI. Voltage maxima and minima, line losses, tap operations, and voltage variability are quantified on a real California distribution feeder. Also, in light of the result of [10], we use a VVC curve with and without a 
deadband to determine the effect on this feeder.

In section [II-A, we introduce the distribution feeder model, relevant definitions, and simulation parameters. In section II-B we introduce the control scheme used for the SI. In section III. we discuss the results of quasi-steady state time series simulations. Finally we conclude the work in IV

\section{Methodology}

\section{A. simulation setup}

PV penetration is well accepted term in literature to describe the ratio of PV to load on a feeder. Here, PV penetration is defined as:

$$
P V_{\text {pen }}=P V_{k V A_{\text {Installed }}} / \text { load }_{\text {peak }} .
$$

$P V_{\text {pen }}$ is fixed for a given feeder configuration. To quantify the importance of solar power generation at a given time instant, we also define instantaneous PV penetration as

$$
P V_{P e n_{\text {Instantaneous }}}=P V_{k V A}\left(t_{i}\right) / \operatorname{load}\left(t_{i}\right) .
$$

Here, we introduce the SI fraction $\left(F r a c_{\mathrm{SI}}\right)$, to describe the ratio of PV capacity that has SI functionality to the total amount of PV on the feeder

$$
\operatorname{Frac}_{S I}=P V_{k V A_{\mathrm{SI}}} / P V_{k V A_{\text {total }}} .
$$

Figure 1 visually depicts a SI fraction of $50 \%$ on the distribution feeder simulated. The PV systems with SI were randomly selected in sequence until $49.5 \%$ capacity was reached. If a selected PV system caused the total capacity to exceed $51 \%$, the entire process was repeated.

Since the feeder is long at $11.7 \mathrm{~km}$ to the furthest bus, voltage regulators exist at the substation and in the middle of the feeder. As a result, the substation voltage setpoint is set to 0.99 p.u.. The feeder is simulated with a total of $107 \mathrm{PV}$ systems, with two of the systems being larger than $0.5 \mathrm{MW}$.

Individual generation profiles were created for each PV system. The profiles were generated using a sky imager following the methodology introduced in [3]. A single timeseries shape scaled according to the maximum power of the load was used for every load in the circuit.

Table I summarizes the simulation setup used in this work.

TABLE I: QSTS simulation setup

Distribution Feeder Simulation Period

PV Penetrations (\%)

SI Fraction $(\%)$

Evaluation Metrics

Simulation software

Load data

PV data

Rural California feeder

95 days $(12 / 10 / 2014-3 / 15 / 2015)$

$0,5,10,15,25,50,75,100,125,150,175,200$

$0,50,100$

Voltage, line losses, tap operations, fluctuations

OpenDSS, Matlab

30 second, scaled substation load profile

30 second, sky image projections

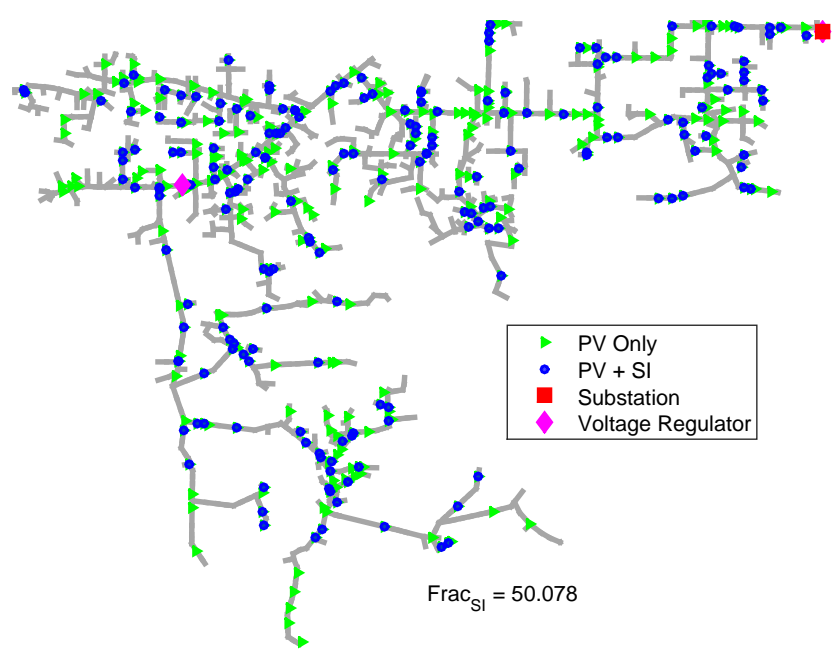

Fig. 1: California feeder with 50\% Smart Inverter fraction.

\section{B. Inverter Control}

[6] introduces a handful of schemes for voltage regulation using SI. However the literature review in section $\mathbb{1}$ showed Volt/VAr control to be an extremely effective approach. Furthermore, VVC is desirable as it prioritizes active power output, meaning no active power is sacrificed to regulate voltage. As a consequence, the amount of inductive or capacitive VArs the inverter can use are restricted by the amount of active power being generated $\left(Q=\sqrt{S^{2}-P^{2}}\right)$.

Because of this, the ratio of inverter $\mathrm{AC}$ power rating to the DC rating of the PV panel becomes important. Generally in practice, $\mathrm{PV}$ inverters are undersized $(\mathrm{AC} / \mathrm{DC}$ ratio $\approx 0.8$ ) to increase energy conversion efficiency. However, the largest PV impacts often coincide with high active power output. Therefore, undersized inverters will not be able to provide sufficient reactive power for voltage regulation. Although SI sizing has not been investigated in the literature, [6] showed that an AC/DC ratio $=1.1$ was sufficient to achieve a constant power factor of 0.9. In this work, all SI are slightly over-sized with an AC/DC ratio of 1.05 .

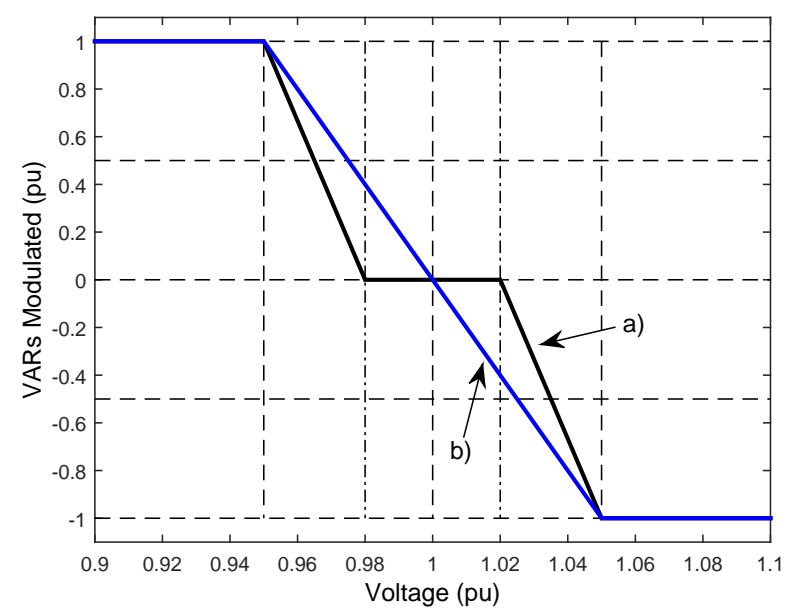

Fig. 2: Control curves used in QSTS. Curve a) has a deadband, while curve b) does not. 
Two VVC curves are investigated in this work, which are given in figure 2. Curve a) has an low voltage limit of 0.95, where it produces the maximum amount of available capacitive VArs, and an upper limit of 1.05 where it produces the maximum amount of available inductive VArs. The curve also exhibits a deaband of 0.04 p.u. between 0.98 and 1.02 p.u., i.e. there is no reactive power support for voltages in that range. Curve b) has the same upper and lower voltage limits as curve a), but has no deadband.

\section{RESULTS}

\section{A. Voltage-Distance Profiles}

Without PV, or at low PV penetrations, voltage decays with distance away from the substation (Figure 3a). Even at such low PV penetration, SI are able to raise bus voltages and counteract voltage decay.

However, as PV penetration increases (Figure 3b), larger voltages are observed at the end of the feeder as a result of large active power outputs. This scenario is observed in figure $3 \mathrm{~b}$ by the $\mathrm{Frac}_{S I}=0$ case, where end of the feeder voltage exceeds 1.06 p.u.. For this day, all SI scenarios drop the end of the feeder voltage below the ANSI limit of 1.05 p.u. The effectiveness of the SI is seen to increase with increasing Frac $_{S I}$ and removal of the deadband. In fact, the curve without the deadband is capable of decreasing end of feeder voltage, while simultaneously increasing voltages near the substation, creating a flatter voltage profile than any of the other scenarios.

\section{B. Maximum Voltage}

The maximum voltage of the feeder over the 95 days simulation period was recorded and is given for each PV penetration in figure 4. In all cases the maximum voltage increases with increasing PV penetration, as expected. At all PV penetrations, the SI with VVC curve b) reduce the maximum voltage, whereas the SI operating under curve a) do not begin to have an effect until the minimum voltage is outside of the deadband range, which occurs first at $P V_{\text {pen }}=75 \%$.

For PV penetrations greater than $150 \%$, the feeder without SI exceeds the ANSI 1.05 p.u. limit. All SI scenarios are able to mitigate the over-voltage condition at $P V_{\text {pen }}=150 \%$. However, Frac $_{\mathrm{SI}}=50 \%$ exhibits violations at $P V_{\text {pen }}>$ 150\%, while both Frac $_{\mathrm{SI}}=100 \%$ scenarios experience violations at $P V_{\text {pen }}-200 \%$. The reason that the SI are not able to mitigate the over-voltage is due to the fact that VVC gives active power priority as discussed in section II-B. Since the maximum voltage occurs when PV generates near its DC rating, the SI does not have sufficient VAr capacity to effectively mitigate the overvoltages.

\section{Minimum Voltage}

The voltage of the feeder recorded over the 95 days experiences a minimum of 0.916 p.u.. Since the minimum voltage occurs during a period of high load and zero PV output, it is not a function of PV penetration. However, as observed in Fig. 5, the effectiveness of SI to mitigate the under-voltage

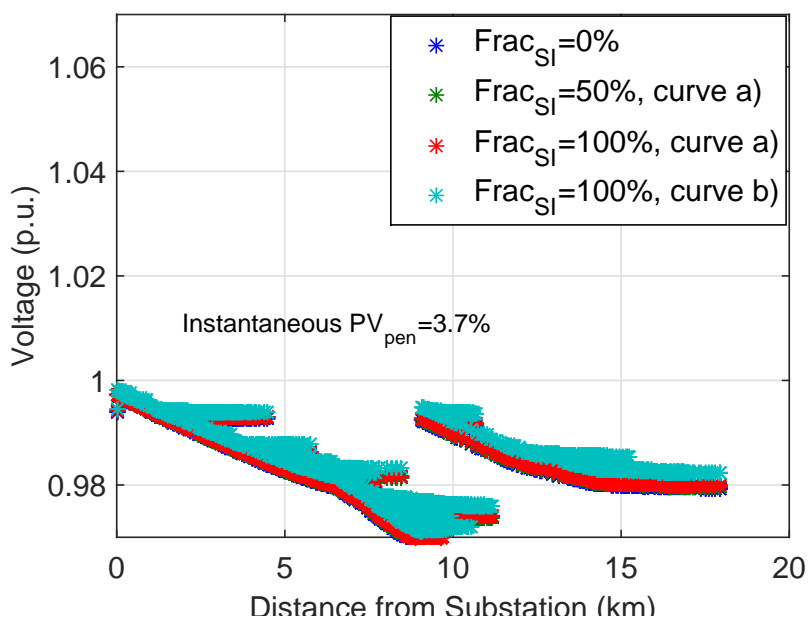

(a)

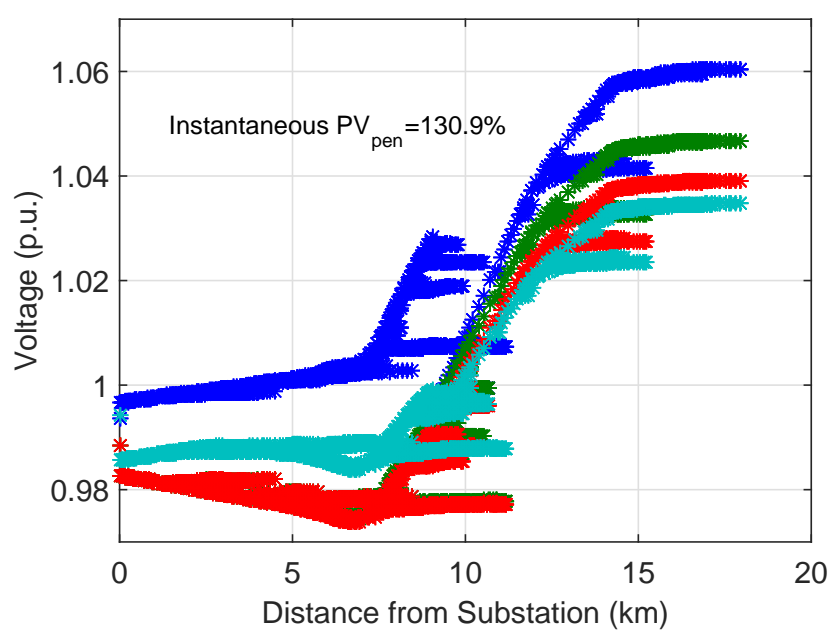

(b)

Fig. 3: Feeder voltage profile as a function of distance for 1/3/2015 12:00:00 PST. (a) $5 \%$ nominal PV penetration. (b) 200\% nominal PV penetration. The curves in each plot represent different SI fractions and VVC curves simulated.

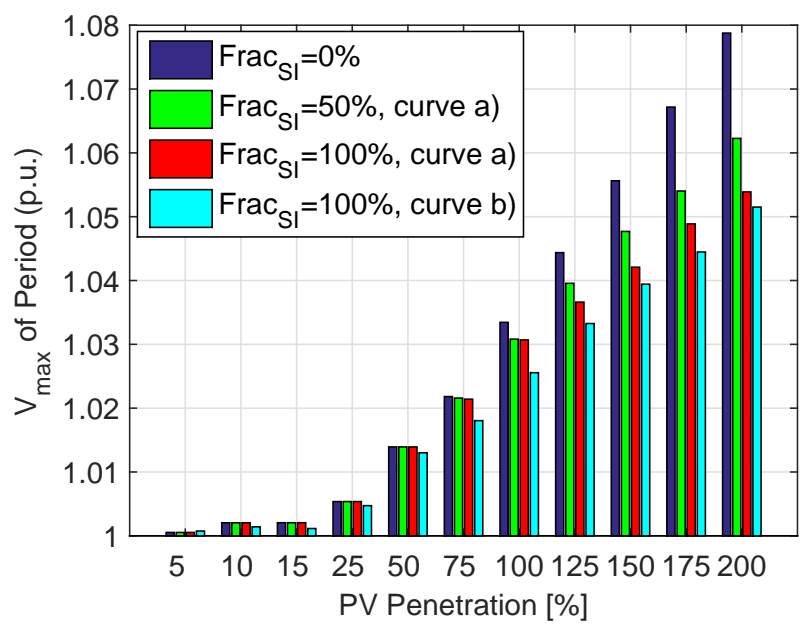

Fig. 4: Maximum voltage recorded on feeder during the 95 day simulation period. The maximum voltage is given for each SI scenario and each PV penetration. 
increases with increasing PV penetration. As PV penetration increases, the aggregate amount of available VArs on the feeder increases, thus giving more control of the voltage. Higher Frac $\mathrm{SI}$ and the removal of the deadband lead to the highest minimum feeder voltages.

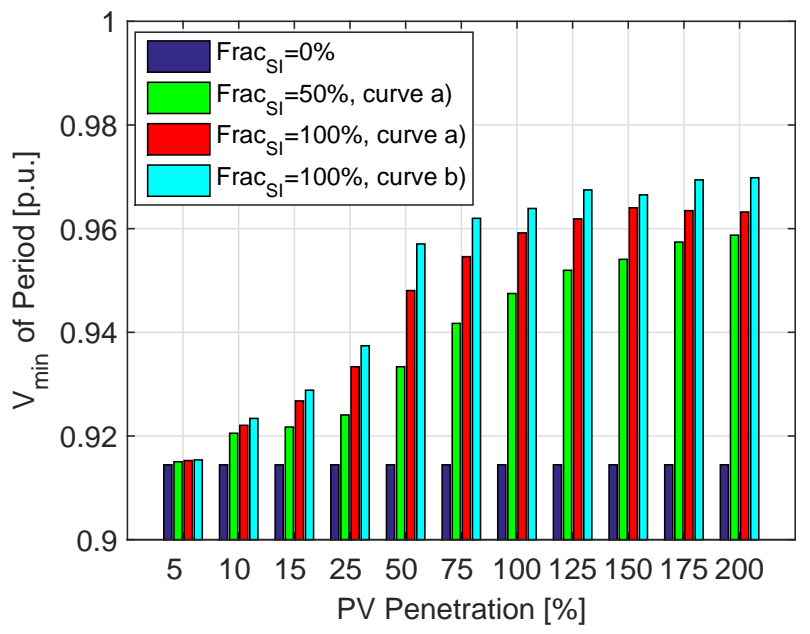

Fig. 5: Minimum voltage recorded on feeder during 95 day simulation period. The minimum voltage is given for each SI scenario and each corresponding PV penetration.

\section{Tap Operations}

The right $y$-axis of Fig. 6 shows that on this feeder the number of tap operations increases from 3.1 tap operations at $5 \%$ PV penetration to 7.3 tap operations per day at $200 \%$ PV penetration. The left $y$ axis indicates that the greatest reduction in tap operations occurs for increasing rrac $_{S I}$ and removal of the deadband, but all SI scenarios reduce the tap operations substantially. In fact, for $\mathrm{frac}_{S I}=100 \%$ and VVC curve b), the SI reduce the tap operations per day to zero. It is expected this trend would continue for higher $P V_{\text {pen }}$, if the SI were over-sized more to allow for sufficient VAr support. This result supports the notion that SI - if properly sited - could act as primary voltage regulators on the circuit.

\section{E. Line Losses}

The normalized line losses in the feeder are given in figure 7. Initially $\left(P V_{\text {pen }}<25 \%\right)$ line losses are reduced for all SI scenarios. However, with increasing PV penetration all SI scenarios eventually produce greater line losses than the feeder without SI. The knee of the curve occurs at $P V_{\text {pen }}=$ $25 \%, 50 \%$, and $75 \%$ and the feeder starts producing line losses greater than the no SI case at $P V_{\text {pen }}=75 \%, P V_{\text {pen }}=125 \%$, and $P V_{\text {pen }}=190 \%$ for $\mathrm{Frac}_{S I}=100 \%$ curve b), $\mathrm{Frac}_{S I}=$ $100 \%$ curve a), and Frac $_{S I}=50 \%$, respectively. These results indicate that the increase in line losses is proportional to the effectiveness of the SI control scheme.

At a high level the reason for increased line losses can be explained by the fact that the SI mitigate over-voltage through inductive VAr support. Since the voltage at the bus then decreases, the current in the line increases and the $I^{2} R$ losses increase.

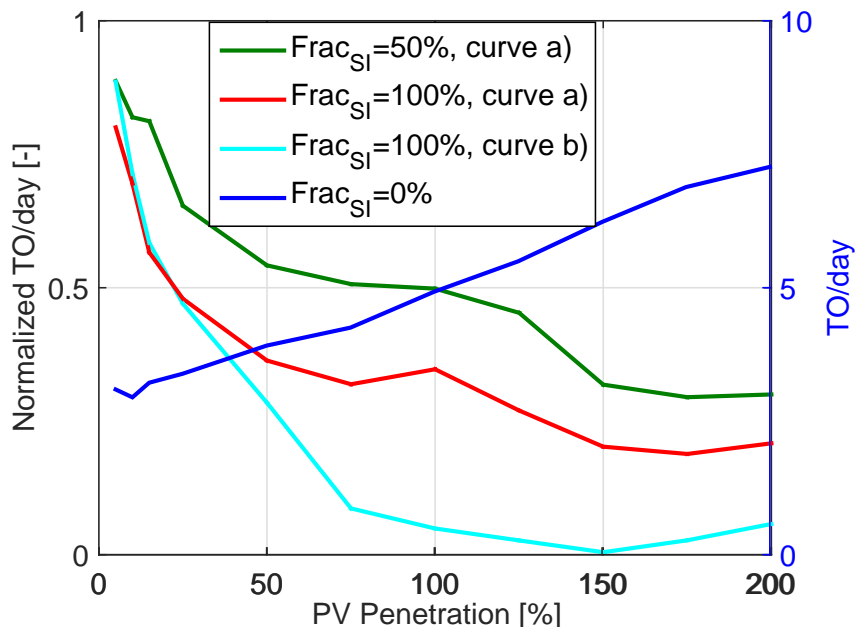

Fig. 6: Tap operations as a function of PV penetration. The plot has a left and right $y$-axis. The right sided $y$-axis gives the average number of tap operations for the feeder without SI as a function of PV penetration. The left sided axis shows the normalized tap operations on the feeder for the different SI cases, where the normalization is performed with respect to the case without SI

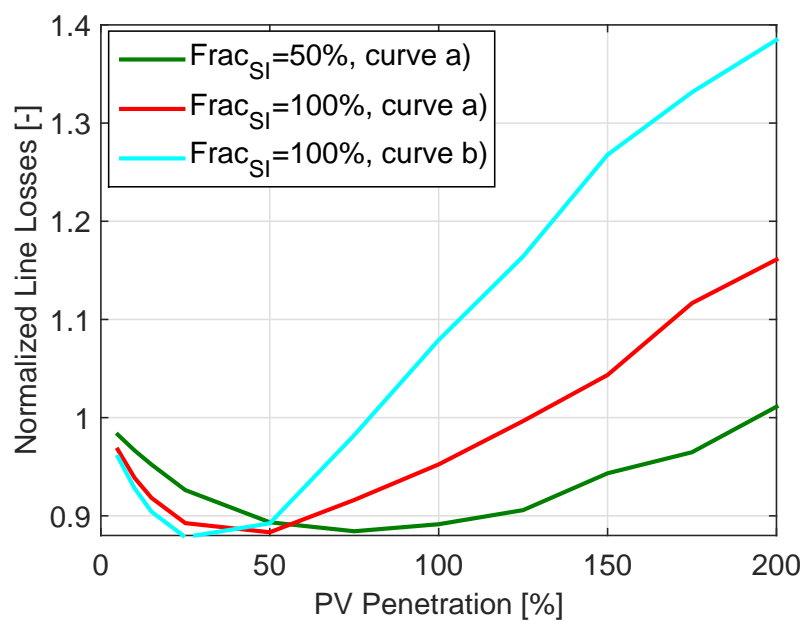

Fig. 7: Normalized power losses in feeder lines as a function of PV penetration. for each SI scenario. The normalization is with respect to the feeder without SI.

\section{F. Voltage Fluctuations}

Voltage fluctuations are often distinguished in two separate types, which have varying effects. Small fluctuations induce power-line flicker manifested through dimming and brightening of lights, but may be too small, localized, or short-lived to impact tap operations. Larger fluctuations cause tap/capacitor operation and can harm behind-the-meter equipment. To capture both types of voltage fluctuations, the solar variability characterization method of [12] is extended to measure voltage fluctuations.

The idea is to assign a variability score (VS) to each feeder configuration over the 95 days. Essentially, the method weights the probability of fluctuations by magnitude, with respect to a set threshold, $V_{0}$. The greater the fluctuations relative to the 
limit, the higher the VS assigned to the feeder configuration.

$$
\begin{gathered}
\widetilde{V}_{\Delta t}=1 / \Delta t\left(\sum_{t}^{t+\Delta t} V-\sum_{t-\Delta t}^{t} V\right) \\
V S_{\widetilde{V}}(\Delta t)=100 \max \left[\mathrm{V}_{0} \times \mathrm{P}\left(\left|\widetilde{\mathrm{V}}_{\Delta t}\right|>\mathrm{V}_{0}\right]\right)
\end{gathered}
$$

The VS is calculated by generating a cumulative distribution function (CDF) of voltage fluctuations $P\left(\left|\widetilde{V}_{\Delta t}\right|>V_{0}\right.$ across all buses for the 95 simulation days. Figure 8 plots the VS for each SI configuration normalized by the VS of the case with 0\% SI. For all SI scenarios and PV penetrations, the variability decreases compared to the case without SI. As observed in the other metrics, the effectiveness of the SI increases with increasing $\mathrm{Frac}_{S I}$ and by removing the deadband. Furthermore, it is observed that the SI improve the VS more and more up to $P V_{\text {pen }}=125 \%$, at which point the effectiveness decreases.

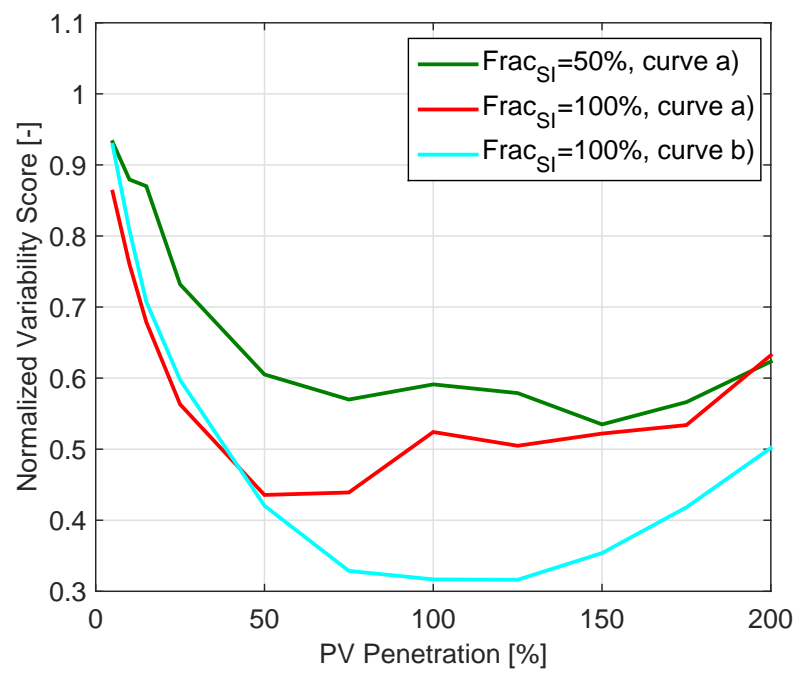

Fig. 8: Normalized VS of each SI scenario as a function of PV penetration.

\section{CONCLUSIONS}

In this work we have simulated a distribution feeder with increasing PV penetration. Varying amounts of smart inverters (SI) operating under different Volt/VAr control schemes were simulated to quantify the effect of SI on the feeder under increasing PV penetration. By observing the effect of SI on voltage (as a function of distance, maximum, and minimum), tap operations, line losses, and fluctuations the following conclusions were drawn.

- SI effectiveness increases with increasing $\mathrm{Frac}_{S I}$.

- Linear Volt/VAr control outperforms the deadband curve.

- Line losses increase with increasing improvements in voltage.

- $\mathrm{AC} / \mathrm{DC}$ ratio is the limiting factor for voltage support.

\section{ACKNOWLEDGMENT}

The authors would like to thank the team at SunSpec and Matt Rylander of EPRI for the expertise they lent on this work. This work was funded by CEC grant PON-14-303.

\section{REFERENCES}

[1] M. ElNozahy and M. Salama, "Technical impacts of gridconnected photovoltaic systems on electrical networksa review," Journal of Renewable and Sustainable Energy, vol. 5, no. 3, p. 032702, 2013.

[2] A. Standard, "C84. 1-1982," American National Standard for Electric Power System and Equipment-Voltage Ratings $(60 \mathrm{~Hz})$.

[3] A. Nguyen, M. Velay, J. Schoene, V. Zheglov, B. Kurtz, K. Murray, B. Torre, and J. Kleissl, "High pv penetration impacts on five local distribution networks using high resolution solar resource assessment with sky imager and quasi-steady state distribution system simulations," Solar Energy, vol. 132, pp. 221-235, 2016.

[4] "Ieee standard conformance test procedures for equipment interconnecting distributed resources with electric power systems - amendment 1," IEEE Std 1547.1a-2015 (Amendment to IEEE Std 1547.1-2005), 2015.

[5] "Generating facility interconnections" california electric rule 21."

[6] B. Seal, "Common functions for smart inverters, version $3, " 2013$.

[7] J. Smith, W. Sunderman, R. Dugan, and B. Seal, "Smart inverter volt/var control functions for high penetration of pv on distribution systems," in Power Systems Conference and Exposition (PSCE), 2011 IEEE/PES. IEEE, 2011, pp. 1-6.

[8] M. J. Reno, R. J. Broderick, and S. Grijalva, "Smart inverter capabilities for mitigating over-voltage on distribution systems with high penetrations of pv," in 2013 IEEE 39th Photovoltaic Specialists Conference (PVSC). IEEE, 2013, pp. 3153-3158.

[9] J. Seuss, M. J. Reno, R. J. Broderick, and S. Grijalva, "Improving distribution network pv hosting capacity via smart inverter reactive power support," in 2015 IEEE Power \& Energy Society General Meeting. IEEE, 2015, pp. 1-5.

[10] S. Abate, T. McDermott, M. Rylander, and J. Smith, "Smart inverter settings for improving distribution feeder performance," in 2015 IEEE Power \& Energy Society General Meeting. IEEE, 2015, pp. 1-5.

[11] I. Kim, R. G. Harley, R. Regassa, and Y. D. Valle, "The effect of the volt/var control of photovoltaic systems on the time-series steady-state analysis of a distribution network," in Power Systems Conference (PSC), 2015 Clemson University, March 2015, pp. 1-6.

[12] M. Lave, M. J. Reno, and R. J. Broderick, "Characterizing local high-frequency solar variability and its impact to distribution studies," Solar Energy, vol. 118, pp. 327$337,2015$. 